\title{
PRESENTACIONES ORALES EN IFE (INGLÉS PARA FINES ESPECÍFICOS): UN ESTUDIO DE CASO EN INGENIERÍA MECÁNICA
}

\author{
Oral Presentations in ESP (English for Specific Purposes): a Case Study in Mechanical \\ Engineering
}

Apresentações orais em IFE (Inglês para Fins Específicos): um estudo de caso em Engenharia Mecânica

\author{
Cristina Gómez Castro (1)
}

Marlisa Amanda Richters (2)

(1) Universidad de León, España. Teléfono: +34 987291774. Correo electrónico: cristina.gomez@unileon.es

(2) Universidad de León, España. Teléfono: +34 987295430. Correo electrónico: mrich@unileon.es

\section{Resumen}

La competencia de saber transmitir información específica relativa a un ámbito de conocimiento de forma oral se presenta como fundamental para cualquier alumno de grado universitario. La experiencia que aquí se presenta se basa en el uso de presentaciones orales en el grado de Ingeniería Mecánica de la Universidad de León en la asignatura de inglés. Desde un punto de vista constructivista del aprendizaje, los alumnos llevaron a cabo una actividad que fue evaluada por medio de una rúbrica y de una presentación de ensayo con feedback. Asimismo, un cuestionario inicial y otro final permitieron observar cómo la percepción del alumnado de este tipo de actividad cambió de forma considerable, valorándola de forma muy positiva tanto para el aprendizaje de la lengua como sobre todo en la consecución de la competencia.

Palabras clave: Presentaciones orales; competencia; IFE; evaluación formativa

\begin{abstract}
Being able to transmit specific information related to a field of knowledge orally is a fundamental competence for any university student. The experience presented here is based on the use of oral presentations in the English course of the degree of Mechanical


Engineering at the University of León. From a constructivist point of view of learning, the students carried out an activity that was evaluated by means of a rubric, and a practice presentation with feedback. Furthermore, an initial and a final questionnaire allowed the authors to observe how students' perception of this type of activity changed considerably. They evaluated it in a very positive way both regarding learning of the language and more especially with respect to the attainment of competence.

Keywords: Oral Presentations; Competence; ESP; formative assessment

\section{Resumo}

A competição de saber transmitir informações específicas sobre um campo de conhecimento por via oral es apresentada como essencial para qualquer grau universitário. A experiência aqui apresentada baseia-se o uso de apresentações orais em Engenharia Mecânica pela Universidade de León na disciplina de Inglês. A partir de uma visão construtivista da aprendizagem, os estudantes realizaram uma atividade que foi avaliada através de uma rubrica e de um ensaio de apresentação com feedback. Também, um questionário inicial e outro final permitiram observar como a percepção dos alunos neste tipo de atividade mudou consideravelmente, avaliando a actividade positivamente para a aprendizagem de línguas e para alcançar a competência.

Palavras-chave: Apresentações orais; competência; IFE; avaliação formativa

\section{Introducción}

Con la entrada en funcionamiento del Espacio Europeo de Educación Superior en España, la enseñanza terciaria ha visto un cambio gradual pero significativo en la implicación del alumnado en su propio aprendizaje y en el escenario mismo de aprendizaje, puesto que, en cualquier titulación, todo profesional ha de adquirir una serie de competencias (Ramos, Álvarez \& Luque, 2010). Entre las competencias generales del Grado en Ingeniería Mecánica de la Universidad de León figura la de "hablar bien en público" así como que los estudiantes "puedan transmitir información, ideas, problemas y soluciones a un público tanto especializado como no especializado”1. Las presentaciones orales se constituyen por tanto en un pilar fundamental a la hora de

\footnotetext{
${ }^{1}$ Véase Memoria de verificación del título disponible en el siguiente enlace: http://seguimiento.calidad.unileon.es/descargas/Mecanica20110429-143-7-28-2011-09-22-18-21-24.pdf Presentaciones orales en IFE (inglés para fines específicos): un estudio de caso en ingeniería mecánica
} 
la consecución de dichas competencias por parte del alumnado y por esta razón se han integrado como parte de la evaluación de la asignatura de idioma inglés en este grado: entre sus ventajas, se encuentra el hecho de que "they help develop important general competencies connected to investigating and solving problems, learning autonomously, investigating, and learning to communicate and cooperate” (ibid, p. 336).

\section{Contextualización}

La experiencia que aquí se describe fue llevada a cabo con los alumnos de primer curso del Grado en Ingeniería Mecánica de la Universidad de León en el contexto de la asignatura obligatoria “Inglés” y durante el curso académico 2015-16. Dicha asignatura cuenta con una carga lectiva de 6 ECTS y el número de alumnado que participó en la experiencia fue la totalidad del matriculado en la misma, 84 alumnos.

La asignatura denominada "Inglés” ha de ser cursada por todos los alumnos del grado y tiene la consideración de obligatoria en un contexto de Espacio de Educación Superior donde la movilidad del estudiantado y su capacidad para poder expresarse en otro idioma se presenta como uno de los pilares básicos, razón que justifica su ubicación en la titulación tal y como se propone en el Libro Blanco de la misma elaborado por la ANECA en 2005.

\section{Diseño y desarrollo}

Como parte de la evaluación de la asignatura, se propuso al alumnado la realización de una actividad consistente en una producción escrita y una producción oral a posteriori basada en la primera, ambas en lengua inglesa. Las especificaciones de la actividad fueron entregadas al alumnado por escrito y también le fueron explicadas en clase, de manera que pudiesen resolverse cuantas dudas les surgieran a los alumnos. Al tratarse de un grado de orientación técnica, se pidió a los alumnos que diseñaran un aparato de su invención con el fin de solucionar algún tipo de problema de la vida cotidiana, en el que tuvieran que definirlo, describirlo y especificar las partes de las que estuviera compuesto, así como indicar su funcionamiento. Dicha tarea debía realizarse en grupos de tres alumnos y ser entregada de forma escrita en primera instancia. Tras recibir feedback de dicha actividad, los alumnos debían llevar a cabo una presentación oral basada en los aspectos más importantes de su invención. Para ello, recibieron clases magistrales con explicación acerca de los aspectos más importantes de las presentaciones orales en general y en particular en una lengua extranjera. Antes de 
llevar a cabo la presentación final, realizaron una presentación de ensayo donde recibieron feedback de parte del profesorado que fue incorporado a la hora de hacer la presentación final, en un proceso de construcción del resultado final.

\section{Evaluación}

Con el fin de evitar que la evaluación de las presentaciones orales fuese "an inaccurate task in which the teacher just gives a mark based on a general impression, without following any reliable criteria” (Ramos, Álvarez, \& Luque, 2010, p. 336) en esta experiencia se utilizó una rúbrica ${ }^{2}$ como instrumento de evaluación de forma conjunta con un ensayo de presentación previo a la presentación final. Dicha rúbrica fue elaborada por el profesorado teniendo en cuenta los aspectos indicados a los alumnos como más relevantes en una presentación oral ${ }^{3}$.

Tabla 1.

Rúbrica de valoración de las exposiciones orales en inglés.

TEACHER \& STUDENT REVIEW - Reviewer's Name: DATE:

Rate each speaker on the following scale of 1 to 5: 1 very poor - 2 poor - 3 ok - 4 good - 5 very good

\begin{tabular}{|l|l|l|l|}
\hline \multicolumn{1}{|c|}{ CRITERIA } & $\begin{array}{c}\text { SPEAKER 1 } \\
\text { Name }\end{array}$ & $\begin{array}{c}\text { SPEAKER 2 } \\
\text { Name }\end{array}$ & $\begin{array}{c}\text { SPEAKER } \\
\text { 3 Name }\end{array}$ \\
\hline $\begin{array}{l}\text { 1. Audibility + Tone/Energy } \\
\text { Can you hear clearly? Varied voice tone? }\end{array}$ & & & \\
\hline $\begin{array}{l}\text { 2. Pace + Fluency } \\
\text { Reading? Too fast? Too slow? Pauses? Hesitations? }\end{array}$ & & & \\
\hline $\begin{array}{l}\text { 3. Eye contact +body language } \\
\text { Across the audience? Confident? Distracting? }\end{array}$ & & & \\
\hline $\begin{array}{l}\text { 4. Use of English (vocabulary, grammar, syntax...) } \\
\text { 5. Pronunciation } \\
\text { Individual sounds; Accent; Comprehensibility; Intonation } \\
\text { Rhythm + stress }\end{array}$ & & \\
\hline $\begin{array}{l}\text { 6. Content Structure/Cohesion } \\
\text { Outline? Signposting? Timing? }\end{array}$ & & \\
\hline $\begin{array}{l}\text { 7. Visual aids } \\
\text { Easy to follow? Support oral speech? }\end{array}$ & & \\
\hline
\end{tabular}

Answer to question(s):

\footnotetext{
${ }^{2}$ Aunque, tal y como indica Pérez-Pueyo (2016, p. 212) una Escala de valoración hubiese sido un instrumento más ajustado para la evaluación de este tipo de actividades, al tratarse en este caso de una asignatura y por lo tanto de una actividad en un segundo idioma, se optó por el uso de una rúbrica con cinco niveles de logro puesto que el alumno debía llevar a cabo la evaluación en un idioma que no es su lengua nativa, añadiendo este aspecto una dificultad a tener en cuenta que podía actuar como condicionante al realizar la evaluación entre iguales.

${ }^{3}$ No se implicó al alumnado en la realización de la rúbrica debido a que, por medio del cuestionario inicial, se pudo comprobar su escaso conocimiento de los aspectos más relevantes a tener en cuenta en una presentación oral.

Presentaciones orales en IFE (inglés para fines específicos): un estudio de caso en ingeniería mecánica
} 
Este instrumento de evaluación fue utilizado tanto por el profesorado como por el alumnado (coevaluación) en las dos fases de la presentación oral (la de ensayo y la final). Asimismo, junto con ella, se emplearon dos cuestionarios, uno al comienzo de la experiencia y otro al final de la experiencia, con el fin de poder recoger las impresiones de los estudiantes sobre presentaciones orales antes de llevarlas a cabo y una vez hubieron realizado la actividad. Dicho cuestionario fue adaptado a partir del utilizado por Gallardo y Martínez en su estudio de $2015^{4}$.

\section{Conclusiones}

Una vez se hubo llevado a cabo la evaluación de la actividad y basándonos tanto en las calificaciones de los estudiantes a lo largo de la misma como en sus respuestas a los cuestionarios, podemos afirmar que la práctica reflexiva y la evaluación formativa han sido claves en el desarrollo de la actividad y en la consecución de una mejor valoración de las presentaciones orales. Al comienzo de la experiencia, un 70\% del alumnado no tenía en cuenta la mayor parte de los parámetros que se evaluaron en sus presentaciones orales, pero una vez hubieron trabajado en ellas y recibido feedback sobre las mismas, más del $80 \%$ coincidieron en que les habían ayudado a mejorar varios aspectos lingüísticos muy importantes como son la pronunciación y la gramática y sobre todo a ser conscientes de la importancia de presentar bien contenidos específicos de su carrera de forma oral ante una audiencia, competencia básica para su futuro tanto académico como profesional (Corredor \& Romero, 2008, p.60).

\section{Referencias}

ANECA (2005). Libro Blanco de Titulaciones de Grado de Ingeniería de la Rama Industrial .Madrid: ANECA.

Corredor, J., \& Romero, C.A. (2008). Planeación, Planeación, organización y expresión de un tipo de discurso oral: la exposición. Consideraciones, sugerencias y recomendaciones. Cuadernos de Lingüística Hispánica, 12, 57-76.

Gallardo del Puerto, F, y Martínez Adrián, M. (2015). The use of oral presentations in Higher Education: CLIL vs. English as a foreign language. Pulso. Revista de Educación, 38, 73-106.

Grussendorf, M. (2007). English for Presentations. Oxford: Oxford University Press.

\footnotetext{
${ }^{4}$ Nuestro agradecimiento a ambos autores por su permiso para utilizar el cuestionario. 
Pérez-Pueyo, A., Hortigüela Alcalá, D., \& Gutiérrez García, C. (2016). Reflexión sobre la evaluación en la formación inicial del profesorado en España. En búsqueda de la concordancia entre dos mundos. Infancia, Educación y Aprendizaje (IEYA), 2 (2), 39-75.

Pérez-Pueyo, A. (2016). El estilo actitudinal en Educación Física: evolución en los últimos 20 años. Retos, 29, 207-215.

Ramos Álvarez, M. \& Luque, G. (2010). A Competence-based Constructivist Tool for Evaluation. Cultura y Educación, 22 (3), 329-344.

Williams, E. J. (2008). Presentations in English. Find your Voice as a Presenter. Oxford: Macmillan. 